\title{
The sensitivity and specificity of mid-upper arm circumference compared to body mass index in screening malnutrition of adult HIV patients taking ART; evidence from selected facilities of Addis Ababa, Ethiopia
}

\author{
Tesfaye Abrhame ${ }^{1}$, Jemal Haidar ${ }^{2}$ \\ ${ }^{1}$ Addis Ababa University, Medical Faculty, School of Public Health, Addis Ababa, Ethiopia \\ ${ }^{2}$ School of Public Health, Addis Ababa University, P.O. Box 27285/1000 \\ Email address: \\ tesfakal.2009@gmail.com (T. Abrhame),hjemal@gmail.com (J. Haidar)
}

\section{To cite this article:}

Tesfaye Abrhame, Jemal Haidar. The Sensitivity and Specificity of Mid-Upper Arm Circumference Compared to Body Mass Index in Screening Malnutrition of Adult HIV Patients taking ART; Evidence from Selected Facilities of Addis Ababa, Ethiopia. Science Journal of Public Health. Vol. 3, No. 1, 2015, pp. 19-24. doi: 10.11648/j.sjph.20150301.14

\begin{abstract}
Introduction: For nutritional assessment of HIV-positive adults and adolescents, it is recommended to take Weight, Height and MUAC (for pregnant and lactating women and/or adults who cannot stand straight) in every contact, such measurements increase the waiting time and inconvenience(1). So that a simple and yet useful anthropometric measurement is needed to screen malnutrition for an immediate nutrition support in developing countries. Objective: To assess the sensitivity (SN) and specificity (SP) of mid-upper arm circumference (MUAC) in detecting under nutrition of adult people with HIV who have a follow up at ART clinic. Methods: A cross-sectional study was conducted from January to July 2013 among adults with HIV (PLHIV), who have follow-up in three selected Governmental Hospitals of Addis Ababa city administration. A total of 594 study subjects were selected using a multi stage cluster sampling technique. MUAC, Height and weight were measured for all subjects and BMI was calculated as $\mathrm{Wt} / \mathrm{Ht}^{2}$. Receivers operating characteristic curve analyses were undertaken to discover the most suitable cut-off values of MUAC for both Men and Women. Results: The prevalence of chronic energy deficiency (CED) based on BMI (BMI $<18.5$ ) was $39.4 \%$ for males and $24.21 \%$ for females, while based on MUAC $<230 \mathrm{~mm}$ for male was $48.4 \%$ and $<220 \mathrm{~mm}$ for female was $35.7 \%$. The sensitivity and specificity of MUAC for Female with optimal criteria of $\leq 224$ for females were 92.86 and 87.07, whereas for Males MUAC with optimal criteria of $\leq 234$ were 94.85 and 68.46 , respectively. The area under curve (AUC) was 0.93 $(p<0.001)$. A MUAC value of $\leq 239 \mathrm{~mm}$ was identified as the best cut off-points to identify CED (BMI $<18.5)$ with SN and SP of $96.43 \%$ and $72.62 \%$, respectively. The PPV was $56.6(95 \% \mathrm{CI}=89.9$ to 99.3$)$ with the highest NPV $98.2(95 \% \mathrm{CI}=66.8$ to 77.9), thus having the highest Youden Index of 0.21. A MUAC value of $\leq 242 \mathrm{~mm}$ was identified as the best cut off point to identify CED (BMI < 18.5) with SN and SP of $96.91 \%$ and $58.39 \%$, respectively. The PPV was $46.3(95 \% \mathrm{CI}=91.2$ to 99.4$)$ with the highest NPV $98.1(95 \% \mathrm{CI}=50.0$ to 66.4$)$, thus having the highest Youden Index of 0.32. Conclusion: a MUAC value of close to $242 \mathrm{~mm}$ for male and $239 \mathrm{~mm}$ for female seemed to be appropriate as a simple and efficient cut-off point for the determination of under nutrition in adult PLHIV.
\end{abstract}

Keywords: Sensitivity, Specificity, BMI, CED, Mid-Upper Arm Circumference, Nutritional Status, ROC Curve

\section{Introduction}

Inadequate nutrition in patients living with HIV (PLHIV) can put them at high risk of chronic diseases particularly if combined with other adverse lifestyle behaviors, received recognition of policy makers and launched national nutritional programs to combat the threat of malnutrition(2).
For individual assessment of body composition and size, anthropometry is being replaced by more accurate but also more complicated methods. Calculation of individual BMI from weight and height still remains a standard and valid tool for the assessment of nutritional status(3),though the complexity of calculations for health workers without mathematical backgrounds or access to calculation software 
is an issue and its use in clinical practice may mask important weight changes and result in a failure to alert healthcare staff to a nutritional problem(4). Furthermore, charts and tables may not be available for those working in traditional health/nutrition services which are often poorly equipped(5).

Currently the national Guidelines for HIV/AIDS and Nutrition in Ethiopia recommends weight, height and MUAC (for pregnant and lactating women and/or adults who cannot stand straight) measurements to be taken in every contact(6) and this entails to increase the waiting time and inconvenience for the patients underlines the need for an alternative anthropometric measurements like Midupper arm circumference (MUAC). MUAC measurement is a reliable and a feasible method of assessment of nutritional status of adults and can be used for screening. An important advantage of using MUAC compared with BMI is that it can be easily obtained in older persons using a simple measuring tape. It has been used for many years as an alternative index of nutritional status in situations like famines and refugee crises, where the height and weight measurements are difficult $(7,8)$. In addition to reliability and simplicity, MUAC demonstrated superior sensitivity to risk of death $(9,10)$, and can offer considerable cost advantages because it only requires measuring tapes which are less expensive than height boards. MUAC measurement is easier to perform on severely malnourished adults than BMI assessment(11)) but no studies have identified the use of MUAC for patient with PLHIV. It is a potentially useful indicator requiring only a cheap, widely available and durable measuring tape that requires no calibration, and having the advantage that it can be measured in patients who have difficulty in standing. Therefore; identification of simple practical ways to assess nutritional status and related outcomes in patients with HIV/AIDS before and during treatment with particular reference to resource-limited settings is mandatory to provide appropriate interventions (12). The purpose of this study was to determine a tool that will be easier and more appropriate for screening of malnutrition among PLHIV and assess the sensitivity and specificity of MUAC in correctly identifying the patient qualifying for supplementary feeding and examine whether BMI can be substituted by MUAC in assessing the nutritional status.

\section{Methodology}

\subsection{Study Design and Settings}

An institution based cross-sectional quantitative study design was employed to assess the sensitivity and specificity of MUAC in Addis Ababa, the capital and largest city of Ethiopia as well as the country's commercial, manufacturing, and cultural center. Its projected population of 2013 is estimated to be 3,038,096 million that resides in 10 sub-cities and 116 Woreda. In the city, there were 36 private and 6 governmental hospitals, 52 health centers (of which 45 are public and 7 are NGO health centres), 136 health stations, 700 private clinics. The numbers of health facilities providing ART services were 66 in number and patients currently on ART were 54,667. The number of ART clients registered in each government hospitals namely Tikur Anbesa specialized (1,302), Saint Paul (987), Zewditu Memorial (1,018), Yekatit 12 (3,065), Ghandi Memorial (987) and Ras Desta Damtew Memorial hospital $(1,011)$ at the time of the study; of these, only three accessible hospitals with large number of clients were purposively selected.

\subsection{Sample Size}

The Sample Size was estimated using single population proportion formula assuming 50\% prevalence of wasting (since there is no information), 5\% degree of precision and 95\% confidence level of certainty and 5\% non response rate with a design effect of (1.5), a total of 594 subjects were estimated and allocated proportionately across the three hospital namely Zeweditu memorial $(\mathrm{n}=345)$; Yekatit 12 ( $\mathrm{n}=135)$; Ras Desta Damtew memorial Hospital $(\mathrm{n}=114)$ The required subjects in each facility were recruited and enrolled consecutively until the required sample size was reached.

\subsection{Data Collection}

Data were collected by trained health workers under the supervision of the PI using anonymous pretested questionnaire containing information on socio-demographic, environmental, anthropometry, dietary. Each completed questioner was checked immediately by the PI to ascertain all the questions have been answered consistently. The information entered was rechecked in $5 \%$ of randomly selected respondents.

Middle upper arm circumference was measured in centimeter with a non-stretched measuring tape with the right arm hanging relaxed. The measurement was taken midway between the tip of the acromion and olecranon process. The tape was placed gently but firmly round the arm to avoid compression of soft tissue. Measurement was taken nearest to $0.1 \mathrm{~cm}$; and subjects were categorized as undernourished when MUAC was $\leq 230 \mathrm{~mm}$ for male and $\leq 220 \mathrm{~mm}$ for females(13).

Weight was measured in kilogram without shoes using a digital bath scale with good precision. Checks on the scale were made routinely before recording the weight of each subject.

Height was taken barefooted in centimeter using stadiometre. A vertical tape fixed perpendicular to the ground on the wall was used as the scale. During the anthropometric measurements the scale was repeatedly checked for accuracy against a known weight. Height was recorded to the nearest $1 \mathrm{~cm}$.

Body mass index (BMI) was calculated as weight in $\mathrm{kg} /$ height in $\mathrm{m}^{2}$, and values below or equal to $18.5 \mathrm{~kg} / \mathrm{m}^{2}$ was considered undernourished or $\operatorname{CED}(14)$ 


\subsection{Data Analysis}

After sorting the data and performed quality control for completeness and consistency, the data were then coded and entered into computers and validated for consistency using EPI version 7.1 and analysis was done using SPSS version 16 for windows. The anthropometric variables were described as mean (SD) and range values. The frequencies of CED were expressed as percentages. Chi-square was utilized to test the significance differences in frequencies of CED among the MUAC categories and BMI and a p-value $<0.05$ was considered statistically significant.

Receiver operating characteristic curve (ROC) analysis was done using Medcalc software version 12.1 to locate the optimal cut-off values of MUAC corresponding to the BMI cut-off in identifying CED cases. Youden index (YI) was calculated as: 'sensitivity + specificity -1 '. Among the different MUAC values, YIs were compared to discern the optimal cut-off points for CED.

\subsection{Ethical Issues}

Ethical approval was obtained from the School of Public Health Research Ethics Review Committee of Addis Ababa University, College of Health Sciences; as well as Addis Ababa Health bureau research ethics committee. Officials of each facility were also contacted, and permission was granted to conduct the study. Informed written consent was obtained from each subject for their participation and the right to withdraw from the study at any time was also communicated to all of them.

\section{Result}

Of the total of 594 respondents, 593 participated making the response rate $99.8 \%$. Over half $(58.5 \%)$ were females. The mean age of study participant was 34 . About one-third $(32.8 \%)$ were single and over half $(51.0 \%)$ were Orthodox. The proportion of respondents who had tertiary level of schooling was $34.7 \%$, and private employees constituted $45.3 \%$ and less than half $(41.7 \%)$ earned less than or equal to 500 Birr, Ethiopian currency, (Table 1).

Table 1. socio-demographic characters of the respondents, Addis Ababa, 2014

\begin{tabular}{lcc}
\hline Characters & Number(N=593) & Percents \\
\hline Sex & 246 & \\
Male & 347 & 41.5 \\
Female & & 58.5 \\
Age(years) & 16 & \\
$\leq 20$ & 53 & 2.7 \\
$21-26$ & 136 & 8.9 \\
$27-32$ & 187 & 22.9 \\
$33-37$ & 118 & 31.6 \\
$38-43$ & 83 & 19.9 \\
$44+$ & $34(7.2)$ & 14.0 \\
Mean (SD) & & \\
Marital status & 195 & 32.8 \\
Single & 231 & 39.0 \\
Married & & \\
\hline
\end{tabular}

\begin{tabular}{lcc}
\hline Characters & Number(N=593) & Percents \\
\hline Divorced & 78 & 13.2 \\
Widowed & 89 & 15.0 \\
Religion & & \\
Orthodox & 304 & 51.3 \\
Protestant & 112 & 18.9 \\
Muslim & 82 & 13.8 \\
Catholic & 64 & 10.8 \\
Educational status & & \\
No schooling & 35 & 5.9 \\
Grade 1-6 & 119 & 20.0 \\
Grade 7-12 & 233 & 39.4 \\
Tertiary schooling & 206 & 34.7 \\
Occupation & & \\
NGO & 62 & 10.5 \\
Government & 139 & 23.4 \\
Student & 30 & 5.1 \\
Housewife & 81 & 13.7 \\
Private/ Merchant & 269 & 45.3 \\
Daily laborer & 12 & 2 \\
Monthly HH Income & & \\
$\leq$ 500 (Birr) & 247 & 41.7 \\
501 - 1,000 & 157 & 26.5 \\
1,001+ & 189 & 31.8 \\
\hline
\end{tabular}

20 Birr=1USD

\subsection{Anthropometric Data}

Table 2 shows the anthropometric measurement of the respondents. The mean (SD) MUAC, weight (SD, height (SD) and BMI (SD) were $161.7( \pm 8) \mathrm{mm}, 56.2( \pm 9.3) \mathrm{kg}$, $242( \pm 31.5) \mathrm{mm}$, and $21.5 \pm 3.4 \mathrm{~kg} / \mathrm{m}^{2}$, respectively.

Table 2. Anthropometric result of the respondents in selected health facilities of Addis Ababa, May 2013.

\begin{tabular}{lcccc}
\hline Variable & Mean & $( \pm$ SD) & Minimum & $\begin{array}{c}\text { Maximu } \\
\mathbf{m}\end{array}$ \\
\hline Height $(\mathrm{cm})$ & 161.7 & 8.1 & 130.0 & 182.0 \\
Weight $(\mathrm{kg})$ & 56.2 & 9.3 & 31.0 & 94.0 \\
BMI $\left(\mathrm{kg} / \mathrm{m}^{2}\right)$ & 21.5 & 3.4 & 16.0 & 32.0 \\
MUAC $(\mathrm{mm})$ & 242.0 & 33.5 & 150.0 & 350.0 \\
\hline
\end{tabular}

$\mathrm{SD}=$ standard deviation

Table 3. Association of MUAC and BMI of the respondents in selected health facilities of Addis Ababa, May 2013

\begin{tabular}{lccc}
\hline & $\begin{array}{c}\text { CED BMI } \\
(\leq \mathbf{1 8 . 5} \mathbf{k g} / \mathbf{m} \mathbf{2})\end{array}$ & $\begin{array}{c}\text { Well nourished BMI } \\
(\mathbf{1 8 . 5} \mathbf{k g} / \mathbf{m} \mathbf{2})\end{array}$ & Total \\
\hline Female MUAC & & & \\
$<220 \mathrm{~mm}$ CED & 92 & 32 & 124 \\
Well nourished & 9 & 214 & 223 \\
Total & 124 & 223 & 347 \\
Male MUAC & & & \\
$<$ 230mm CED & 76 & 43 & 119 \\
Well nourished & 4 & 123 & 127 \\
Total & 80 & 166 & 246 \\
\hline
\end{tabular}

\subsection{Sensitivity and Specificity of MUAC and BMI}

Receiver operating characteristic curve analysis of sensitivity and specificity of MUAC using the cutoff point of $240 \mathrm{~mm}$ and $220 \mathrm{~mm}$ for male and female is computed respectively using Medcalc version 12 Software. The 
22 Tesfaye Abrhame and Jemal Haidar: The Sensitivity and Specificity of Mid-Upper Arm Circumference Compared to Body Mass Index in Screening Malnutrition of Adult HIV Patients taking ART; Evidence from Selected Facilities of Addis Ababa, Ethiopia

sensitivity and specificity of MUAC for Female with optimal criteria of $\leq 220 \mathrm{~mm}$ were $91.9 \%$ and $86.9 \%$, respectively whereas for Males MUAC with optimal criteria of $\leq 230 \mathrm{~mm}$ were $95 \%$ and $74 \%$ respectively.

Receiver operating characteristic curve analysis of sensitivity and specificity of MUAC for Female with optimal criteria of $\leq 226 \mathrm{~mm}$ were $94.9 \%$ and $85.9 \%$ respectively whereas for Males MUAC with optimal criteria of $\leq 234$ were $94.8 \%$ and $68.5 \%$ respectively.

The results of ROC curve analyses for Female MUAC with optimal criteria of $(\leq 226 \mathrm{~mm})$ shows sensitivity $(\mathrm{SN})$ $94.9 \%$, specificity (SP) $85.9 \%$, positive predictive value (PPV) 71.2\% and negative predictive value (NPV) 97.5\% for each MUAC value to identify CED are presented in
(Table 4).

The results of ROC curve analyses of Male MUAC are presented in (Table 6$)$ with optimal criteria of $(\leq 230 \mathrm{~mm})$, with the sensitivity (SN), specificity (SP), positive predictive value (PPV) and negative predictive value (NPV) for each MUAC value to identify CED.

The use of MUAC for female correctly diagnosed $74.2 \%$ of cases of CED and 96\% with normal nutritional status and the use of MUAC for male correctly diagnosed $96.9 \%$ of cases of CED and $63.9 \%$ with normal nutritional status. Similar study done on MUAC correctly diagnosed $82.35 \%$ of cases of CED and $81.82 \%$ of women with normal nutritional status.

Table 4. Receiver operating characteristic curve of MUAC with sensitivity and specificity of female respondents in selected health facilities of Addis Ababa, May 2013.

\begin{tabular}{|c|c|c|c|c|c|c|c|}
\hline Criterion & Sensitivity & $95 \%$ CI & Specificity & $95 \% \mathrm{CI}$ & PPV & NPV & YI \\
\hline$\leq 226 \mathrm{~mm}$ & 94.05 & $86.7-98.0$ & 85.93 & $81.1-89.9$ & 71.2 & 97.5 & 0.135 \\
\hline$\leq 230 \mathrm{~mm}$ & 95.24 & $88.3-98.7$ & 78.33 & $72.9-83.2$ & 61.9 & 97.8 & 0.184 \\
\hline$\leq 231 \mathrm{~mm}$ & 96.43 & $89.9-99.3$ & 77.57 & $72.0-82.5$ & 61.4 & 98.3 & 0.183 \\
\hline$\leq 239 \mathrm{~mm}$ & 96.43 & $89.9-99.3$ & 72.62 & $66.8-77.9$ & 56.6 & 98.2 & 0.219 \\
\hline$\leq 240 \mathrm{~mm}$ & 97.62 & $91.7-99.7$ & 63.88 & $57.8-69.7$ & 50 & 98.6 & 0.277 \\
\hline$\leq 240.5 \mathrm{~mm}$ & 98.81 & $93.5-100.0$ & 63.88 & $57.8-69.7$ & 50.3 & 99.3 & 0.27 \\
\hline
\end{tabular}

Table 5. Estimated specificity at fixed sensitivity of Female respondents in selected health facilities of Addis Ababa, May 2013.

\begin{tabular}{cccc}
\hline \multicolumn{3}{c}{ Estimated specificity at fixed sensitivity } \\
\hline Sensitivity & Specificity & 95\% CI & \multicolumn{1}{c}{ Criterion } \\
\hline 80 & 89.56 & 85.42 to 93.46 & $\leq 221.7467$ \\
90 & 87.83 & 81.90 to 92.02 & $\leq 223.43$ \\
95 & 79.85 & 58.92 to 89.35 & $\leq 229.2$ \\
97.5 & 64.75 & 18.77 to 82.56 & $\leq 239.9$ \\
80 & 94.98 & 88.78 to 98.79 & $\leq 229.12$ \\
90 & 77.92 & 61.77 to 95.34 & $\leq 221.63$ \\
95 & 64.83 & 36.60 to 78.47 & $\leq 219.23$ \\
97.5 & 31.17 & 18.93 to 55.94 \\
\hline
\end{tabular}

Table 6. Results of receiver operating characteristic curve analyses of mid-upper arm circumference with sensitivity and specificity of Male respondents in selected health facilities of Addis Ababa, May 2013.

\begin{tabular}{|c|c|c|c|c|c|c|c|}
\hline Criterion & Sensitivity & $95 \%$ CI & Specificity & $95 \%$ CI & PPV & NPV & YI \\
\hline$\leq 234 \mathrm{~mm}$ & 94.85 & $88.4-98.3$ & 68.46 & $60.3-75.8$ & 52.7 & 97.3 & 0.258 \\
\hline$\leq 239 \mathrm{~mm}$ & 94.85 & $88.4-98.3$ & 66.44 & $58.3-74.0$ & 51.1 & 97.2 & 0.273 \\
\hline$\leq 240 \mathrm{~mm}$ & 96.91 & $91.2-99.4$ & 59.06 & $50.7-67.0$ & 46.7 & 98.1 & 0.316 \\
\hline$\leq 242 \mathrm{~mm}$ & 96.91 & $91.2-99.4$ & 58.39 & $50.0-66.4$ & 46.3 & 98.1 & 0.32 \\
\hline$\leq 244 \mathrm{~mm}$ & 97.94 & $92.7-99.7$ & 58.39 & $50.0-66.4$ & 46.5 & 98.7 & 0.315 \\
\hline
\end{tabular}

Table 7. Estimated specificity at fixed sensitivity of Male respondents in selected health facilities of Addis Ababa, May 2013.

\begin{tabular}{cccc}
\hline \multicolumn{4}{c}{ Estimated specificity at fixed sensitivity } \\
\hline Sensitivity & Specificity & $\mathbf{9 5 \%}$ CI & Criterion \\
\hline 80 & 69.13 & 59.65 to 79.87 & $\leq 230.32$ \\
90 & 69.13 & 61.07 to 76.36 & $\leq 232.55$ \\
95 & 65.89 & 27.52 to 75.17 & $\leq 239.075$ \\
97.5 & 58.39 & 4.70 to 73.15 & $\leq 243.15$ \\
80 & 75.4 & 60.63 to 85.57 & $\leq 228.0941$ \\
90 & 24.74 & 13.22 to 60.82 & $\leq 212.45$ \\
95 & 18.07 & 1.78 to 28.04 & $\leq 209.3062$ \\
97.5 & 2.53 & 0.00 to 22.99 & $\leq 187.525$ \\
\hline
\end{tabular}

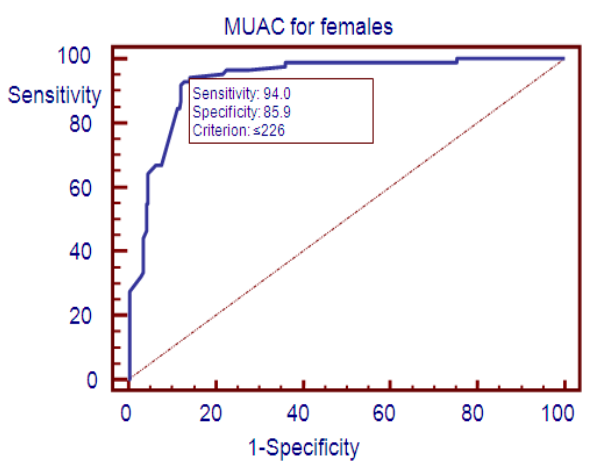

Figure 1. Receiver operating characteristic curve of Female respondents MUAC in selected health facilities of Addis Ababa, May 2013. 


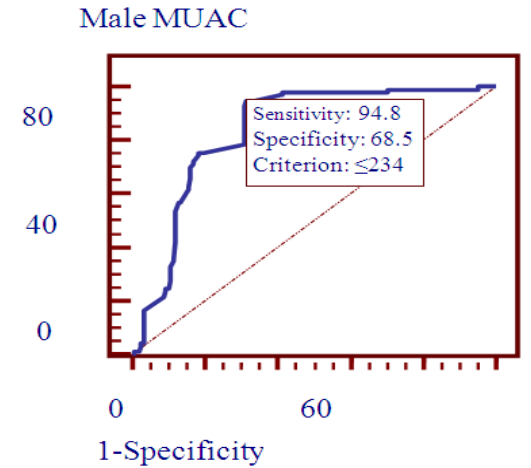

Figure 2. Receiver operating characteristic curve analysis of MUAC of Male respondents in selected health facilities of Addis Ababa, May 2013.

\section{Discussion}

The present study found that thirty five percent of women based on MUAC $(\leq 220 \mathrm{~mm})$ and twenty-nine percent based on BMI (less than $18.5 \mathrm{~kg} / \mathrm{m}^{2}$ ) were Undernourished whereas forty-eight percent of men based on MUAC $(\leq 230 \mathrm{~mm})$ and thirty-two percent based on BMI were undernourished. Since the study was aimed at assessing the sensitivity and specificity of MUAC in detecting under nutrition of PLWH. The determination of an adult population's Nutritional Status is recognized as of prime importance when assessing for population health and wellbeing. There are different modalities for this, BMI is most often used and MUAC is also recognized as a useful and simpler tool for screening adult individuals for poor nutritional status(15) and has been shown to accurately reflect adult nutritional status as defined by BMI(16).

The prevalence of CED (BMI $\leq 18.5$ ) was $32.5 \%$ and $29.1 \%$ for male and female, were as the prevalence of under nutrition based on (MUAC $\leq 230 \mathrm{~mm} \&$, $\leq 220 \mathrm{~mm}$ ) were $48.4 \%$ and $35.7 \%$ in male and female respectively. The sensitivity and specificity of MUAC for Female and Male with optimal criteria of $\leq 220 \mathrm{~mm}$ and $\leq 230 \mathrm{~mm}$ were 91.9\% and 95\% respectively whereas for Males MUAC with optimal criteria of $\leq 234 \mathrm{~mm}$ were $94.8 \%$ and $68.4 \%$ respectively. The sensitivity and specificity of MUAC for Female and male with optimal criteria of $\leq 224 \mathrm{~mm}$ were $92.8 \%$ and $87.0 \%$, respectively whereas for Males MUAC with optimal criteria of $\leq 234 \mathrm{~mm}$ were $94.8 \%$ and $68.4 \%$ respectively.

There was a significant positive association between MUAC and BMI, regression analysis demonstrated that MUAC had a significant positive relation with BMI; the percentage of the variation in BMI explained by MUAC was $71 \%$. The binary Logistic regression analysis demonstrated that overall, $82.2 \%$ of cases of CED were correctly classified by using MUAC. The use of MUAC for female correctly diagnosed $74.2 \%$ of cases of CED and $96 \%$ with normal nutritional status and the use of MUAC for male correctly diagnosed $96.9 \%$ of cases of CED and $63.9 \%$ with normal nutritional status this finding is similar with other studies done on MUAC which correctly diagnosed $82.4 \%$ of cases of CED and $81.82 \%$ of women with normal nutritional status.

The present study demonstrated a significant positive correlation between $\operatorname{MUAC}$ and $\operatorname{BMI}(r=.71, p<0.01)$. MUAC value of $239 \mathrm{~mm}$ was found to be most appropriate in identification of female subjects with CED, and a value of $242 \mathrm{~mm}$ was most useful in identifying men with CED. Both of these values are higher than the internationally recommended men's MUAC cut-off value of $230 \mathrm{~mm}$ and females $220 \mathrm{~mm}$.

It is also recognized as an effective means of screening for poor NS in adults(17)-(15). However, the recommended MUAC cut-off value of $230 \mathrm{~mm}$ to define in men(3) may not be the most appropriate for all ethnic groups. A study from Nigeria(18) reported that a MUAC cut-off point of $230 \mathrm{~mm}$ was optimal for the north of the country, while a $240 \mathrm{~mm}$ Cut-off point was more appropriate for the south. Thus, there is a need to establish ethno-specific MUAC cutoff points. Similarly, a cut-off point of $240 \mathrm{~mm}$ was reported to be suitable in a recent study from the south of India(19); however, a recent study(20) of non-tribal adult slum dwellers of Bengalee ethnicity in West Bengal, India, reported a MUAC value of $240 \mathrm{~mm}$ to be the most appropriate cut-off point for identifying CED (BMI<18.5). A possible reason for cut-off points being higher than that suggested by James et al(3) is that persons of South Asian origin (eg the Oraon) have higher levels of regional adiposity (irrespective of BMI) compared with other ethnic groups(21)-(22).

Under nutrition is more prevalent in males than females this might be due to the peripheral adiposity of females, Individuals with a MUAC less than $240 \mathrm{~mm}$ were approximately twice as likely to report recent illness compared with those with a MUAC equal to or above 240 $\mathrm{mm}(20)$. Again in this study, MUAC close to $240 \mathrm{~mm}$ was appropriately sensitive to reported illness. However, this study combined with the similar findings discussed, suggests an urgent need to revisit BMI and MUAC cut-off values as the measures of CED and under-nutrition.

The area under curve (AUC) was $0.93(p<0.001)$ (Table 9). A MUAC value of $\leq 239 \mathrm{~mm}$ for female was identified as the best cut off to identify CED (BMI $<18.5 \mathrm{~kg} / \mathrm{m}^{2}$ ) with SN and SP of $96.43 \%$ and $72.62 \%$, respectively. The PPV was 56.6(CI 89.9-99.3) with the highest NPV 98.2 (CI 66.8-77.9), thus having the highest YI of 0.21.

Another result of ROC curve analyses for Male MUAC are presented, with the sensitivity (SN), specificity (SP), positive predictive value (PPV) and negative predictive value (NPV) for each MUAC value to identify CED. The area under the curve (AUC) was $0.83(p<0.0001)$ (Table 11). A MUAC value of $<=242 \mathrm{~mm}$ for males was identified as the best cut off to identify CED (BMI <18.5) with SN and SP of $96.91 \%$ and $58.39 \%$, respectively. The PPV was 46.3(CI 91.2-99.4) with the highest NPV 98.1 (CI 50.066.4 ), thus having the highest Youden Index of 0.32 .

The mean BMI in this study for age group15-49 is 21.85 $\mathrm{kg} / \mathrm{m} 2$ and $21.02 \mathrm{~kg} / \mathrm{m} 2$ for women and men respectively. Forty-two percent of women have a normal BMI (between 
24 Tesfaye Abrhame and Jemal Haidar: The Sensitivity and Specificity of Mid-Upper Arm Circumference Compared to Body Mass Index in Screening Malnutrition of Adult HIV Patients taking ART; Evidence from Selected Facilities of Addis Ababa, Ethiopia

18.5 and $24.9 \mathrm{~kg} / \mathrm{m} 2$ ), while 29 percent of women are thin or undernourished (BMI less than $18.5 \mathrm{~kg} / \mathrm{m} 2$ ) and 7 percent are overweight or obese (BMI $25 \mathrm{~kg} / \mathrm{m} 2$ or above) this finding is supported by other studies except undernourished $27 \%$ (23). Thirty -two percent of men have normal BMI (between 18.5 and $24.9 \mathrm{~kg} / \mathrm{m} 2$ ), while 39.4 percent of men are thin or undernourished (BMI less than $18.5 \mathrm{~kg} / \mathrm{m} 2$ ) and 4.5 percent are overweight or obese (BMI $25 \mathrm{~kg} / \mathrm{m} 2$ or above).

\section{Acknowledgement}

We thank all the study subjects including the staffs of Addis Ababa Health Bureau, Zewditu Memorial Hospital, Ras-Desta Memorial Hospital and Yekatit 12 Hospital for their unreserved support during the data collection process.

\section{Reference}

[1] Ferro-Luzzi A SS, Franklin M, James WPT. A simplified approach to assessing adult chronic energy deficiency. European Journal of Clinical Nutrition 1992;46:173-86.

[2] WHO. Physical status: the use and interpretation of anthropometry. Report of a WHO Expert Committee. World Health Organ Technical Report Series. 1995.

[3] James WP M-TG, Norgan NG, Bistrian BR, Shetty PS, Ferro-Luzzi A. The value of arm circumference measurements in assessing chronic energy deficiency in Third World adults. European Journal of Clinical Nutrition 1994;48:883-94.

[4] Bisai S BK. Undernutrition in the Kora Mudi tribal population, West Bengal, India: a comparison of body mass index and mid-upper-arm circumference. Food Nutr Bull2009;30(1):63-7.

[5] Gustafson VFG, C>S $>$ Vieira, B. Samb, A. Naucler, Predictors for Death in HIV-positive and HIV-negative Tuberculosis Patients in Guinea-Bissau. 2007;35(2):69-80.

[6] EFMOH. National Guidelines for HIV/AIDS and Nutrition in Ethiopia. 2000.

[7] Jelliffe DB JE. The arm circumference as a public health index of protein-calorie malnutrition in early childhood. Journal of tropical pediatrics. 1996;15(177-260.).

[8] Velzeboer Mea. Evaluation of arm circumference as a public health index of protein-calorie malnutrition in early childhood. Joumal of tropical pediatrics1983;29(135-44).

[9] Briend A DC, Graven K, Mazumder RN, Wojtyniak B. Usefulness of nutritional indices and classifications in predicting death of malnourished children. BMJ1986;923:373=5.

[10] Vella V TA, Ndiku J, Marshal T, Cortinovis I Anthropometry as a predictor for mortality among Ugandan children, allowing for socio-economic variables. Eur J Clin Nutr 1994;48:189-97.

[11] Steve Collins M, BS. Using Middle Upper Arm Circumference to Assess Severe Adult Malnutrition During Famine JAMA1996;276(5):391-5.

[12] WHO. Executive Summary of a scientific review. Consultation on Nutrition and HIV/AIDS in Africa.(Evidence, lessons and recommendations for action. Durban, South Africa); 2005.

[13] BAPEN. Malnutrition universal screening tool (MUST); British Dietetic Association, 2011.

[14] Z. Cook SK SLaSS. Use of BMI in the assessment of undernutrition in older subjects: reflecting on practice. 2005;64:313.

[15] T.Khadivzadeh. Mid-upper arm and calf circumferences as indicators of nutritional status in women of reproductive age. Eastern and Mediterranean Health Journal 2002; 8:612-8.

[16] Collins.S. Using middle upper arm circumference to assess severe adult malnutrition during famine. JAMA 1996;276:391-5.

[17] Bose K GS, Mamtaz H, Mukhopadhyay A, Bhadra M. High prevalence of undernutrition among adult Kora Mudi tribals of Bankura District, West Bengal, India. Anthropological Sciences 2006;114:65-8.

[18] AAO. Identification of underweight women by measurement of the arm circumference. International Journal of Gynecology and Obstetrics 1990;31:231-5.

[19] Rodrigues VC RR, Lena A. . Utility of arm circumference as a screening instrument to identify women at nutritional risk. Tropical Doctor 1994;24:164-6.

[20] India Go. Census of India. (Online) 2001. $\mathrm{http}: / w w w c e n s u s i n d i a g o v i n ~ 2010$.

[21] K. B. Generalised obesity and regional adiposity in adult White and migrant Muslim males from Pakistan in Peterborough. Journal of Royal Society of Health 1996;116:161-7.

[22] Deurenberg P YM, van Staveren WA. Body mass index and percent body fat: a meta- analysis among different ethnic groups. International Journal of Obesity Related Metabolic Disorder 1998;22(12):1164.

[23] Calverton M, USA, Central Statistical Agency Ethiopia Ethiopia Demographic and Health Survey 2011. Central Statistical Agency and ICF International2012:152-88. 